\title{
Du Grand Temps à l'a-chronie narrative.
}

\section{Enjeux d'une « connivence des temps » dans La Déchirure}

Si tout en restant moderne, je n'avais pas pris l'habitude d'imaginer la simultanéité de ce temps vécu et du temps que je vis encore avec le temps de la légende qui dépasse le premier - et ce depuis que j'ai appris à parler puis à lire - je n'aurais jamais commencé à écrire de romans, et pour le moins je n'aurais jamais pu continuer dans cette voie.

À considérer la question du temps dans La Déchirure (1966), d'emblée c'est un sentiment de vertige qui assaille le lecteur. Les strates temporelles, mises en jeu à travers les découpages et réassemblements textuels orchestrés par Henry Bauchau, semblent en effet sans fin. Le lecteur se trouve emporté, peut-être même sans s'en apercevoir à la première lecture, par une narration à multiples fils temporels qui s'entrelacent et se chevauchent, dans un effet stylistique de reprise. Ainsi, dans ce roman, l'auteur ne se contente pas de jouer sur la scansion des jours et des nuits qui organise la macrostructure du récit. Cette scansion, qui reprend en partie le tempo originel de la partition de la Genèse biblique, s'ingénie surtout, dans le même temps, à travailler l'écart avec celui-ci, afin que depuis le Grand Temps mythique de cette Genèse fondatrice, le narrateur trouve à rassembler les temporalités éparses de son récit fondateur personnel.

En réalité, il appert en cours de lecture que la temporalité de ce roman laisse paraître, de façon ostensible, les déchirures ${ }^{\mathbf{1}}$ qui l'habitent et qui tendent, ainsi, à rendre le récit littéralement a-chronique ${ }^{2}$, étant donné que les différentes périodes narrées s'entrecroisent et se font écho les unes aux autres selon des modalités analogiques, davantage qu'elles ne se succèdent selon l'ordre de la chronologie factuelle.

1 Pour des développements autres sur la question des déchirures du temps dans l'œuvre bauchalienne, voir mon ouvrage : Henry Bauchau, une écriture en résistance, Paris, L'Harmattan, "Structures et pouvoirs des imaginaires ", 2006, p. 53-70.

2 Voir infra pour les analyses portant sur ce concept dans La Déchirure. 
Comment, alors, la narration de ce roman travaille-t-elle à maintenir ensemble " durée et simultanéité ", selon la formule de Bergson³ ? Et comment cette mise en " concordances " entre des conceptions du principe de temporalité opposées jouet-elle dans le processus de réélaboration du Moi narrateur ? C'est à ces questions qu'entendent répondre les analyses qui suivent.

\section{Du Grand Temps de la Genèse à la genèse de l'écriture}

À l'enseigne de la citation d'Oe Kenzaburo qui ouvre cet article, le premier temps qui inscrit sa marque sur le livre, temps fondateur de l'écriture bauchalienne au même titre que de celle du prix Nobel japonais, se rapporte à celui du mythe, que l'on nommera "Grand Temps " à la suite de Mircea Eliade ${ }^{4}$. La présence de ce temps originaire s'appréhende dans le récit selon deux mouvements distincts qui, in fine, se complètent. Tout d'abord, le temps du mythe trouve une première incarnation à travers un personnage emblématique du récit : la Sibylle, figure hautement ambivalente pour l'écrivain et d'emblée apparentée par lui aux grandes pythies grecques pourvoyeuses de la parole divine, et donc de la parole destinale des êtres. D'ailleurs, cette figure antique se fait jour sous les auspices d'un titre en soi révélateur : "La Sibylle la plus ancienne "(D98, p. 32-37), tandis que, dans le maillage textuel même, références à la mythologie antique et histoire personnelle du narrateur s'enchevêtrent déjà de façon inextricable.

Le chapitre s'ouvre sur l'image du lieu de consultation de "la Sibylle ", aussi appelée "Madame " (D98, p. 34), de façon plus prosaïque : "Il fallait pénétrer, aux environs des Champs-Élysées, dans un grand immeuble [...]" (D98, p. 32, je souligne). En une phrase se conjoignent l'avenue la plus célèbre de Paris, d'une part, et, d'autre part, la sphère la plus profonde des Enfers où se retrouvent les âmes vertueuses après leur mort, une fois qu'elles ont été jugées aptes à y résider. Le fait que la Sibylle habite " un grand immeuble ", si près du paradis grec, permet, malgré la crainte qu'elle suscite chez le narrateur, de lui conserver une part humaine plus rassurante. Cependant, la métaphore de l'antre inquiétant se poursuit quelques paragraphes plus loin, lorsque le narrateur, inquiet de l'avenir de cette rencontre écrit : "On sentait qu'en cas d'échec on pourrait très bien crever là, cette grande caverne à trafics étant un lieu prédestiné pour un fait divers, pour une mort un peu abandonnée, par exemple, dans les lieux d'aisance "(D98, p. 33, je souligne). La " caverne ", lieu où s'énonce - se joue - le " destin », côtoie ainsi " le fait divers " et " les lieux d'aisance " du XX $\mathrm{X}^{\mathrm{e}}$ siècle.

3 Henri Bergson, Durée et simultanéité. À propos de la théorie d'Einstein (1922), Paris, PUF, "Quadrige ", 2009.

4 Pour plus de détails, voir Mircea Eliade, Mythes, rêves et mystères (1957), Paris, Gallimard, "Folio essais ", 1989. Le mythologue souligne, notamment, que "le mythe est censé exprimer la vérité absolue, parce qu'il raconte une bistoire sacrée, c'est-à-dire une révélation trans-humaine qui a eu lieu à l'aube du Grand Temps, dans le temps sacré des commencements (in illo tempore) ». (p. 21, souligné dans le texte.) 
Le second versant de ce Grand Temps s'affiche dans la structure de l'ouvrage donnée en table des matières. En effet, ce n'est pas pur effet d'optique si, en consultant celle-ci, on est amené à effectuer un rapprochement avec la temporalité initiale d'une création bien plus fabuleuse : celle du monde dans la Genèse biblique.

L'ordonnancement de l'ouvrage s'organise en effet aux mêmes points d'articulation : les jours et les nuits qui précèdent le septième jour, celui du repos. Cependant, les altérations, évidentes et essentielles dans le processus créateur de Bauchau, permettent, tout en creusant l'écart avec la Genèse biblique, de laisser poindre suffisamment de ressemblances pour interpeller le lecteur. Ainsi, la scansion des jours et des nuits, articulant l'architecture d'ensemble du livre bauchalien, suit de façon presque mimétique - mais tout repose en ce presque - celle de la création du monde par Dieu. En d'autres termes, c'est selon cette architecture narrative que s'inscrit l'empreinte du Grand Temps, nécessaire à la genèse du récit bauchalien dans le temps profane.

Henry Bauchau ne se prend certes pas pour Dieu, mais lorsque l'on garde en mémoire la crise de foi religieuse que l'écrivain a traversée dans les années 50, il n'est pas interdit de penser que c'est en partie ${ }^{5}$ cette crise qui autorise une prise de liberté à l'égard de la lettre de la Genèse, afin de la subvertir partiellement. La distance prise vis-à-vis de sa première ferveur chrétienne permet ainsi à l'écrivain, à travers ces jeux de miroir à l'égard de la structure du texte biblique, de donner corps d'écriture à sa propre genèse et, en d'autres termes, de donner naissance à une ouvre qui lui donne corps d'écrivain.

Il est important de souligner d'emblée en quoi l'interprétation du récit de $L a$ Déchirure en tant que genèse personnelle, se confirme dans l'ouvrage lui-même, et ce à travers la déconstruction que fait subir l'écrivain à la structure narrative biblique. Ainsi, le Grand Temps mis à contribution par l'œuvre bauchalienne ne fait pas seulement appel aux figures de l'antiquité grecque mais aussi, dans un même mouvement, à plusieurs figures de la Bible. La plus explicite est celle de Moïse qui s'incarne, momentanément, dans le grand-père aidant les siens à traverser l'incendie de la ville, au début de la Première Guerre mondiale. Le rapprochement est opéré par Bauchau lui-même dans le chapitre "L'incendie de Sainpierre ":

Je le [le grand-père] vois très bien, le visage et la barbe noircis, les yeux qui pleurent sous le lorgnon, au point de paraître saigner. Il a dû jeter son chapeau de paille qui prenait feu et il se protège avec sa veste dont les manches nouées autour de la tête le font ressembler à Moïse. (D98, p. 46)

La convocation explicite de la figure de Moïse vient renforcer le sentiment du lecteur, qui a pu pressentir dans la configuration temporelle du récit inscrite en table des matières une connivence avec le texte biblique. Bien que tenue à distance,

5 Il faut noter à cet égard que les poèmes de jeunesse restés inédits s'avèrent d'emblée en distance à l'égard de la doxa chrétienne. 
d'une part, cette connivence, reste tout de même entretenue par l'écrivain à travers l'écriture, d'autre part. Ainsi, de même que la chronologie de la Genèse est détournée par le roman, la figure héroïsée de Moïse, sauveur de son peuple et le guidant vers la terre promise - notamment grâce au miracle de la traversée de la mer rouge -, se trouve ramenée à une dimension quelque peu démythifiée dans une mise en miroir thématique inversée. Certes le grand-père s'apparente à une figure de héros, puisqu'il traverse une ville en flammes - par opposition avec son prédécesseur qui, lui, fait traverser les flots à son peuple. Cependant, le patriarche bauchalien, bien qu'il sauve sa famille - version amoindrie du peuple guidé par Moïse -, à ce moment du récit, entraîne celle-ci avec lui dans une sorte d'exode sans point ultime d'arrivée, sans terre promise comme horizon d'attente - je reviendrai plus loin sur un autre passage de "l'incendie de Sainpierre "6.

Dès lors, sollicitée par ces deux allusions bibliques, la curiosité du lecteur ne manque pas de chercher à suivre la direction qu'elles suggèrent en l'étendant à l'ensemble du roman lui-même : d'autres figures bibliques feraient-elles l'objet d'autres jeux de palimpseste dans La Déchirure?

Pour les enjeux que cherche à soulever cet article sur la question du temps dans le roman, il n'est pas besoin de dresser un inventaire exhaustif des rapprochements possibles avec la Bible en général, ni la Genèse en particulier. Cependant, deux exemples, implicites ceux-là, méritent que l'on s'y attarde, car ils mettent en relief en quoi les transferts d'un texte à l'autre participent à l'élaboration de l'a-chronie narrative - je développe plus loin cette notion -, par nouement de ces éléments appartenant au Grand Temps avec ceux du temps profane, déchiré, qui informent le récit bauchalien.

Dès «Le premier jour ", alors que le narrateur introduit le personnage de la psychanalyste sous les traits mystérieux et fascinants d'une pythie grecque, surgissent soudainement, à travers le même personnage, d'autres figures féminines éloignées de la civilisation grecque, mais essentielles pour la Bible : Ève et Marie. Celles-ci ne sont pas explicitement nommées mais, accompagnées de l'animal maudit entre tous par Dieu, le serpent, leur présence se fait jour en sous-main :

Ce qu'il aurait fallu interpréter [...] [c’était aussi les évolutions froides, la courbe fascinante d'un serpent. On découvrait une rapidité, une souplesse, une opacité d'un autre âge qui était le serpent et une plénitude de chaleur qui était la femme. Le serpent était écrasé sous le talon de la femme mais il avait rependu sa semence au pied de l'arbre et la terre était imprégnée de sa mobilité séduisante. (D98, p. 34-35)

La Genèse, dans la traduction de Louis Segon (1910), qui est la plus répandue dans la jeunesse d'Henry Bauchau, dit précisément :

6 Pour d'autres analyses sur le chapitre "L'incendie de Sainpierre », voir : Isabelle Vanquaethem, « La remémoration de la guerre 1914-1918 chez Henry Bauchau : l'écriture de l'incendie de Sainpierre ", dans Textyles. Revue des lettres belges de langue française, n 32-33 : "14-18: Une mémoire littéraire ", 2007, p. $127-142$. 
3.14 L'Éternel Dieu dit au serpent : Puisque tu as fait cela, tu seras maudit entre tout le bétail et entre tous les animaux des champs, tu marcheras sur ton ventre, et tu mangeras de la poussière tous les jours de ta vie.

3.15 Je mettrai inimitié entre toi et la femme, entre ta postérité et sa postérité : celle-ci t'écrasera la tête, et tu lui blesseras le talon.

Par transfert, les liens du récit bauchalien au temps de l'immémorial se révèlent : par superposition des figures féminines convoquées sous la plume de l'écrivain la Sibylle, Ève, la Vierge Marie, la figure de la psychanalyste ${ }^{7}-$, ce sont trois temporalités qui s'entrecroisent inextricablement dans le texte (le temps de la mythologie antique, celui de la Bible et le temps profane du roman). La multiplication de ces connexions indique ainsi clairement que le Grand Temps imprime sa marque dès l'œuvre fondatrice de Bauchau. De même, le détournement par superposition de figures symboliques déplacées de leurs ancrages respectifs porte à conséquence pour le narrateur, car dans le passage cité du roman, c'est dans le personnage même de la psychanalyste que s'incarnent et le serpent et la figure féminine qui l'écrase. En effet, la suite du texte dit ceci :

La femme existait d'une pensée magnifique, on percevait l'équilibre de ses épaules et, dans la circulation de sa vie, la saveur de la sève et le sel des choses marines. Mais le serpent était le plus ancien, aussi y avait-il parfois un silence, une densité presque insupportable et ce regard sans yeux sur toutes choses, qui était comme un autre sexe, inconnu. (D98, p. 35)

En d'autres termes, la femme qui vainc le serpent finit par se confondre avec lui dans la figure de l'analyste. Dès lors, la recomposition que fait subir l'auteur aux valeurs symboliques du Grand Temps n'en finit pas, dans les jeux de redistributions au cours du récit, de jouer avec - et de se jouer de - leur sens, en leur imprimant un caractère duplice, dont ils se trouvent investis en raison de la suture que l'écriture bauchalienne leur impose avec le temps profane.

Depuis cette reconfiguration du Grand Temps au cœur du récit, une autre figure biblique se fait jour, qui importe d'autant plus que le narrateur identifie celui qu'il était enfant à cette dernière. Cette nouvelle référence s'opère encore dans un écart marqué par rapport au texte source. Il s'agit du motif de la lutte entre David et Goliath, relatée dans le premier livre de Samuel (situé parmi les « Livres historiques »).

J'étais réduit, enfermé entre ses membres, alors il [l'homme noir] s'asseyait sur moi. Un jour, grâce à l'herbe glissante, j'ai pu me dégager, je lui ai lancé une pierre. Il a été atteint au front, il est tombé. Je crois que je me suis mis à pleurer, mais il a ri comme s'il était fier de ma méchanceté. (D98, p. 83)

7 Dans le passage cité du roman le rapprochement opéré entre le serpent écrasé par le pied de la femme et "l'arbre ", au pied duquel le serpent déverse sa semence, semble faire allusion à la scène citée de la Genèse. Le rapprochement avec la figure de Marie s'origine, lui, dans une tradition chrétienne plus tardive, à partir d'une extrapolation du texte de l'Apocalypse (chapitre 12), dans lequel la femme qui s'oppose au dragon est identifiée à la Vierge, mais sans que le texte dise explicitement qu'elle écrase physiquement ce dernier. 
Cette scène, volontairement élaborée sur le seuil entre onirisme et séance d'analyse - comme la plupart des scènes de ce genre dans le roman - prend place au cours de la première nuit. En effet, les interpositions de ces nuits permettent à l'auteur de suspendre le temps douloureux de l'agonie de la mère afin d'y substituer, le temps "d'une nuit ", ou plus exactement de quelques pages, la progression des séances de thérapie. Ces récits d'enfance et de rêves se rapportent alors à ce temps révolu de la vie du petit garçon que le narrateur a été ; mais aussi, et peutêtre surtout, au temps encore vif de la jeunesse de la mère.

Dans la Bible, David entre en lutte contre son adversaire philistin :

17.42 Le Philistin regarda, et lorsqu'il aperçut David, il le méprisa, ne voyant en lui qu'un enfant, blond et d'une belle figure.

17.43 Le Philistin dit à David : Suis-je un chien, pour que tu viennes à moi avec des bâtons ? Et, après l'avoir maudit par ses dieux,

17.44 il ajouta : Viens vers moi, et je donnerai ta chair aux oiseaux du ciel et aux bêtes des champs.

[...]

17.48 Aussitôt que le Philistin se mit en mouvement pour marcher au-devant de David, David courut sur le champ de bataille à la rencontre du Philistin.

17.49 Il mit la main dans sa gibecière, y prit une pierre, et la lança avec sa fronde; il frappa le Philistin au front, et la pierre s'enfonça dans le front du Philistin, qui tomba le visage contre terre.

17.50 Ainsi, avec une fronde et une pierre, David fut plus fort que le Philistin ; il le terrassa et lui ôta la vie, sans avoir d'épée à la main.

Dans le roman, le passage au niveau profane altère, là encore, la portée héroïque de la scène biblique. En effet, le garçon de la scène romanesque ne tue pas son "Philistin " avec sa pierre, mais se contente de le faire tomber. Cela lui permet, au moins, de gagner cette bataille contre celui qui est seulement identifié par le syntagme "l'homme noir " dans le roman. L'indice le plus significatif de l'altération du statut héroïque du jeune garçon se révèle notamment dans le rire qu'éveille chez "l'homme noir " la surprise de la réussite de l'attaque qu'il vient de subir.

Ces références discrètes dans le cours de l'œuvre permettent d'inscrire, toujours sous les auspices de la figure tutélaire de la Sibylle, que le temps profane (conventionnellement divisé en passé, présent et futur) ne peut prendre corps dans ce livre qu'en relation étroite avec ce Grand Temps immémorial et fondateur. Et l'intérêt pour le lecteur réside dans le fait que la force intrinsèque du récit puise à la " concordance des temps ${ }^{\mathbf{8}}$ narratifs par discordances des temps narrés.

8 Pour reprendre le titre d'un article de Henry Bauchau, sur lequel je reviens plus loin : " La connivence des temps ", dans EE, p. 107-119. 


\section{Des lambeaux de temps à l' $a$-chronie narrative}

Si l'on se rapporte à la table des matières de l'ouvrage, la chronologie semble plutôt limpide. Pourtant, une lecture attentive fait apparaître que deux aspects singuliers de la chronologie de cette semaine posent problème. D'une part, cette dernière ne contient que six jours et non sept ; d'autre part, deux nuits manquent à l'appel de la logique chronologique de l'ensemble.

Sur le premier point, il semble possible d'envisager l'absence de septième jour comme l'effacement du jour de repos du Dieu créateur. Dieu ayant fini son ouvrage prend le temps d'une journée où il ne fait rien, autrement dit où il ne se passe rien. La Genèse biblique fait place à ce septième jour au coeur même de sa narration, le jour de repos divin fait donc partie intégrante de la création. C'est parce que la création est finalisée que le créateur peut se reposer et qu'il confère un statut particulier à ce jour pour les humains. Or, si la chronologie de La Déchirure semble suivre la succession temporelle d'une semaine complète - le dimanche en moins -, il s'avère, en réalité, que dans l'agencement de ces journées dans le texte, le septième jour de la Genèse correspond au cinquième jour du roman ; celui qui suit le décès de la mère du narrateur. Ainsi se poursuit la déconstruction de la chronologie, puisque le " premier jour " du récit n'est pas en adéquation avec le " premier jour " de la Genèse. En d'autres termes, le jour manquant dans le récit bauchalien en vient à recomposer le rythme même de la semaine du calendrier chrétien. Par suite, l'illusion première du bel ordonnancement des faits vole en éclats.

Le passage de la Genèse au monde profane, impose ainsi, du même geste, le passage du Grand Temps au temps historique. Selon une logique similaire à celle de la suppression du septième jour, l'effacement des deux dernières nuits du récit semble, là encore, participer d'une démythification des enjeux temporels. Cette disparition semble s'expliquer à travers le statut particulier de la quatrième nuit au cour du récit ; celle où le décès de la mère fait basculer le livre. On note déjà un fort déséquilibre de vitesse de récit entre les jours et les nuits qui se situent avant et après cette nuit fatidique : le récit occupe plus de 200 pages avant "la dernière nuit " (D98, p. 251-263), alors que les deux derniers jours n'occupent eux qu'une dizaine de pages. Comme si le temps, après "la dernière nuit ", s'affolait. Par ailleurs, les nuits qui précèdent la dernière sont toutes dédiées au temps de l'analyse et du travail des rêves, et opèrent donc comme des segments extradiégétiques par rapport à la ligne narrative première. Comme je l'ai signalé plus haut, cela permet à l'écrivain de suspendre la narration de l'agonie maternelle et, dans le même mouvement, de montrer les différentes étapes de l'évolution intérieure du narrateur par rapport à son enfance et à ses transformations intérieures. Et ce, quand bien même les séances d'analyse et les rêves sont rapportés dans un ordre imitant l'aléatoire.

En fait, le découpage narratif de l'œuvre, qui oscille en permanence entre récit diégétique et extradiégétique, s'ingénie davantage à rapporter ces événements par 
rapprochements ana-logiques, plutôt que selon la loi de l'ordre chrono-logique. Et les intertitres qui scandent les journées et les nuits participent de cette construction hors chronologie, puisqu'ils sont insérés afin de perturber l'enchaînement causal des faits. Il semble ainsi que l'écart organisé vis-à-vis de la structure du récit de la Genèse entend, dès le seuil du roman, signaler que les lacunes temporelles de la macrostructure narrative ne manqueront pas d'avoir une incidence toute particulière sur la microstructure du récit elle-même. En d'autres termes, les découpes et redistributions des différentes époques narrées dans La Déchirure, savamment orchestrées par l'auteur, visent à saper l'autorité d'un temps parfaitement linaire et strictement successif entre passé, présent et futur, dont l'image la plus célèbre est sans doute la flèche du temps.

Cette stratégie de reconstruction narrative, par dissémination et réassemblage des temps narrés, rapproche l'écrivain de l'un des fondateurs du genre autobiographique - et grand interrogateur de la question du temps -, Saint Augustin. L'un des passages essentiels des Confessions de l'homme d'Église permet de souligner combien la pensée d'une multiplicité des présents - du passé, du présent et de l'avenir -, qui nous hantent continuellement, fonde le soubassement de l'architecture de La Déchirure.

Ce n'est pas user de termes propres que de dire : " il y a trois temps, le passé, le présent et l'avenir. " Peut-être dirait-on plus justement : "il y a trois temps, le présent du passé, le présent du présent, le présent du futur. " Car ces trois sortes de temps existent dans notre esprit et je ne les vois pas ailleurs.?

Ainsi, le déplacement opéré par Bauchau du niveau macrostructurel du texte à sa microstructure va bien au-delà de l'usage habituel des jeux de renvois analeptiques et proleptiques définis par Gérard Genette ${ }^{\mathbf{1 0}}$. L'écrivain, en effet, ne s'est pas contenté d'inscrire des sauts temporels d'un paragraphe à un autre, il use aussi souvent, dans le cours même des paragraphes, de l'enchaînement syntactique afin de faire glisser le lecteur d'une temporalité à une autre de façon plus subreptice, ce qui participe à l'égarement de ce dernier dans les replis du texte. Ainsi en va-t-il, par exemple, dans l'enchaînement de deux temporalités distinctes au cour de la scène qui suit :

La fumée [de l'incendie de Sainpierre], naturellement, on l'avait oubliée. Olivier découvrait toujours ces choses, si évidentes, que personne ne les voyait plus. Il y avait donc la fumée, celle d'une ville qui brûle avec tous ses meubles et tous ses greniers. C'est à cause de cette fumée que j'ai toujours une vision confuse de la réalité et que je distingue mal, à travers le hublot de l'avion l'étendue pourtant considérable des inondations. (D98, p. 37-38)

9 Saint Augustin, Les Confessions (XI, 20), trad. Joseph Trabucco (1964), Paris, Flammarion, "GF ", 1979 , p. 269.

10 Gérard Genette, Figures III, Paris, Seuil, "Poétique ", 1972 ; repris dans Discours du récit, Paris, Seuil, "Points-Essais ", 2007, p. 78. Cette deuxième édition sert ici de référence. 
Ce passage introduit le début du second paragraphe de la partie intitulée "L'incendie de Sainpierre ». Il constitue un préambule au texte cité plus haut, qui offre une remémoration de l'incendie fondateur de la première blessure d'enfance. En fait, tout le récit de l'incendie est inventé, réélaboré par le narrateur, ce dernier étant trop jeune au moment des faits pour pouvoir s'en rappeler. Cependant, l'enchaînement syntactique montre de façon éclatante le procédé employé : à l'instar du mot "pression ", qui faisait passer à rebours et par analogie, d'une époque à une autre, d'une scène d'attente de train - temps du présent narré -, à celle de l'attente d'un train dans l'enfance - temps du présent de narration ${ }^{\mathbf{1 1}}$-, dans le cas présent, c'est le motif "fumée " qui fait passer, au creux de la phrase même, d'une scène à l'autre. Et - ironie volontaire ? - le lecteur passe, du même geste, de l'incendie structurellement fondateur pour le narrateur aux " inondations " qui rendent le paysage aussi marécageux et informe que son esprit peut l'être en ce point du récit.

Ces rapprochements ana-logiques opèrent le plus souvent, au-delà des parties scandées par les intertitres mentionnés, par saut de paragraphe, et sont pour une part visualisables grâce à trois astérisques de séparation, comme l'atteste l'exemple qui suit, première occurrence du fait :

Le lendemain, après la visite du médecin, j'ai commencé à comprendre que je n'avais pas subi une défaillance ou une défaite passagère. J'avais été terrassé et marqué. Pour la vie, et pour l'éternité s'il y en a une. J'étais abattu aux pieds de l'ennemi inconnu et il n'y avait plus de combat possible. C'est mon propre poids qui me retenait la face contre terre, le talon de l'adversaire n'était pas nécessaire. Peut-être ne l'avait-il jamais été. Si le salut était encore possible - et il devait l'être puisque somme toute je paraissais vivant - il ne pouvait plus venir que d'ailleurs et de l'autre. Il fallait de l'aide. Attendre. Pour cela les coups de maillet m'aidaient beaucoup.

La ville approche, je vois le lac recouvert de brume. Je descends vers le déplaisir, la souffrance et une grande privation du cour et des sens. Le train m'emmène vers la gare, un avion, la mécanique est déclenchée. On dirait que j’ai rompu mes amarres et que je dérive vers un autre monde, pour la seconde fois [...]. (D98, p. 29-30)

Dans cet enchaînement, tiré de la première journée, le paragraphe initial termine une longue remémoration d'un événement antérieur de plusieurs années à la semaine du récit diégétique qui a déclenché le " terrassement " auquel fait référence le narrateur, sans datation précise par rapport à la chronologie d'ensemble. Le texte qui suit les trois astérisques de séparation/jointure rembraye sans connecteur logique apparent sur la diégèse, en évoquant le départ du narrateur pour rejoindre sa mère malade.

11 On opère une distinction entre le " présent de narration ", qui peut couvrir n'importe quelle temporalité narrée, et le "présent narré " qui s'applique uniquement au temps narré concordant strictement avec le présent diégétique. Voir Émile Benveniste, "Les relations du temps dans le verbe français ", dans Problèmes de linguistique générale (1966), vol. 1, Paris, Gallimard, "Tel ", 1976, p. 237-255. 
Cependant, les sauts temporels n'opèrent pas tous selon ce principe de séparation évidente. Avant même le passage cité, d'autres glissements chronologiques ont déjà été orchestrés par l'écriture, où ce sont bien davantage les mots et un certain usage de la syntaxe qui rendent possibles les mises en rapport temporelles entre les scènes rapportées. La composition narrative repose ainsi sur la production d'un savant travail de repiquages textuels, depuis les recompositions syntactiques et syntagmatiques des événements narrés. Le passage suivant est, en ce sens, exemplaire de ces jeux entre différents tissus mémoriels :

À la gare le vent est glacial, il est venu un peu de soleil mais qui ne réchauffe pas. Le petit train blanc et bleu paraît tout fripé sur la neige. Il ressemble à un de ces jouets idiots, qui traînent encore au fond d'un placard sans s'apercevoir que l'enfance est finie. J'ai un moment d'effroi devant ces wagons vides. Il faut pourtant que je grimpe dans le compartiment. Que je fasse les gestes nécessaires, avant de m'asseoir et de supporter à nouveau la pression.

La pression qui s'exerçait le dimanche soir à Sainpierre, après la journée de visite aux grands-parents, pendant que nous attendions dans la gare l'arrivée du train. C'était le soir, et particulièrement à l'arrivée formidable des locomotives enveloppées de vapeurs blanches, qu'on éprouvait le mieux le sentiment de n'être pas soi-même [...]. (D98, p. 22)

Deux éléments interviennent dans ce passage, afin de conjoindre les deux parties du texte. D'une part, la reprise du syntagme "la pression " permet de faire suture ${ }^{\mathbf{1 2}}$ entre deux chronologies a priori incompossibles entre elles, puisque disparates dans la logique des événements narrés. D’autre part, la concomitance des deux espaces distants chronologiquement offre, dans le même temps, le jeu d'une mise en miroir puisque, dans les deux situations évoquées, le narrateur se retrouve dans une gare, en attente d'un train. Zone de transit, la gare est aussi un seuil, lieu inhabitable, lieu par excellence dans lequel le narrateur ne peut manquer de se sentir dépris de lui-même : sans ancrage référentiel d'identification à lui-même. Et ce qui vaut pour l'aspect spatial de la gare vaut pour le niveau temporel. Sans ancrage spatial, impossible pour le narrateur de s'ancrer dans un temps historiquement déterminé. D’où un usage fréquent du " on ", plutôt que du "je » dans l'ensemble du récit. À l'instar de ce que dit la suite du texte : "C'était le soir, et particulièrement à l'arrivée formidable des locomotives enveloppées de vapeurs blanches, qu'on éprouvait le mieux le sentiment de n'être pas soi-même. Il y avait un tremblement, un doute fondamental, douloureux - mais alors avec plaisir - ou délicieux, jusqu'aux limites de l'écourement, sur sa propre identité et la véracité des choses réelles " (D98, p. 22).

12 Pour reprendre le terme sous une autre forme, qui file la métaphore de la couture, on peut dire aussi que le texte "fait pression " entre les deux paragraphes à travers le syntagme même "la pression ", au sens où l'on parle d'un "bouton pression " que l'on utilise afin de joindre ensemble deux parties d'un vêtement. 
Ce qui provoque le glissement d'une gare vers l'autre, c'est le sentiment de dépossession de soi que revit le narrateur du fait de l'agonie de la mère. Il renvoie ce dernier à la sensation d'éparpillement intérieur qu'il a de lui-même. L'écriture bauchalienne joue subtilement, à travers les raccords qu'elle performe, de ces désancrages temporels et d'identités : il n'est pas possible au lecteur de décider duquel procède l'autre. Est-ce la déconstruction temporelle qui engendre celle du Moi narrateur, ou l'inverse ? L'intrication de l'une dans l'autre au fil du récit laisse cette question sans réponse assurée.

Par suite de ce dessaisissement de l'être et de l'ordre chrono-logique, quand bien même les récits d'enfance entament leur narration durant "le premier jour " du récit, ils ne s'y cantonnent pas, afin de laisser place aux événements postérieurs de la vie du narrateur. Le stratagème, qu'exhaussent les deux passages cités de la première journée, opérant par raccordements textuels et non selon l'ordre factuel de l'histoire, se perpétue en effet jusqu'en fin de volume. C'est pourquoi le récit d'enfance tend à s'inscrire sous le signe de l'intemporel, lui qui modèle encore le narrateur au moment de la rédaction du livre. Ce caractère intemporel du récit d'enfance est lisible de façon emblématique dans le déplacement, vers la fin du volume, d'une scène fondamentale dans la vie du narrateur, qui permet de comprendre le cheminement qui a été le sien jusqu'à la rédaction du livre. Il s'agit de la scène dite de "la circonstance éclatante " qui fera d'ailleurs l'objet, sous ce même titre, d'une analyse plus théorique, vingt ans après la rédaction de La Déchirure.

Cette scène, qui se passe lorsque le narrateur a quatre ou cinq ans n'est rapportée que durant la quatrième journée du récit, après de très longs passages dédiés aux séances d'analyse qui ont elles-mêmes découlé, pour une bonne part, de la rupture qu'opère cette scène d'enfance dans la vie du narrateur (D98, p. 216-217). Dès lors, la narration travaillant davantage par sédimentation de segments narrés, les éléments rapportés de façon chronologique finissent eux-mêmes par être contaminés par cette prolifération non pas ana-chronique de l'histoire contée, mais davantage $a$-chronique.

Sur ce point théorique, mon propos suit la distinction proposée par Gérard Genette entre anachronie et achronie. Sa définition de l'achronie s'avère, en effet, en parfaite adéquation avec la construction narrative dominante mise en place par Henry Bauchau : "Ici, aucune inférence du contenu ne peut aider l'analyste à définir le statut d'une anachronie privée de toute relation temporelle, et que nous devons donc bien considérer comme un événement sans date et sans âge : comme une achronie "13. Il est, de fait, très difficile, à s'en tenir à la trame narrative ellemême - sinon à s'appuyer sur la biographie de l'auteur -, de restituer une chronologie précise à l'ensemble des événements narrés dans le texte.

13 Gérard Genette, Discours du récit, op. cit, p. 78. 
Dans le cas de La Déchirure, le rapport de discordance entre l'ordre de l'histoire et celui du récit, nécessaire à la mise en perspective globale de l'ana-chronie narrative et à sa restitution dans la chronologie générale de l'ouvrage, est particulièrement difficile à établir, voire impossible pour certains segments. Or ce refus d'identification de la chronologie de l'histoire (ou, au moins d'une grande partie de celle-ci) n'est pas fortuit. Les dates sont volontairement rares et réservées à la période de la Première Guerre mondiale (en fait ses dates de début et de fin : 19141918), alors que, par ailleurs, tous les segments temporels, qui forment ainsi des bandelettes de discours quasi autonomes dans la grande machine textuelle, pourraient être rapportés selon un ordre narratif tout autre au cours du récit. La mesure de l'anachronie ne peut ainsi être envisagée pour ce texte ${ }^{\mathbf{1 4}}$, ce qui fait qu'elle perd là de sa signification. L'a-chronie - au sens où la chronologie n'a plus de prérogative sur la scansion textuelle -, semble imposer son tempo à l'œuvre bauchalienne. Et ce travail de l'a-chronie, "que nous devons donc bien considérer comme un événement sans date et sans âge „15, pour reprendre les termes de Gérard Genette, fait ainsi noud avec la figure tutélaire du Grand Temps qui préside, comme on la vu plus haut, à certains événements réels et/ou oniriques insérés dans le récit.

\section{De la «connivence des temps » à la suture du Moi narrateur}

Cette $a$-chronie narrative se trouve renforcée en ses prérogatives sur le texte par deux autres éléments constitutifs de ce dernier. Le premier concerne l'emploi quasi constant dans le récit de ce que la critique littéraire nomme le "présent de narration ".

En effet, l'enchaînement des scènes diégétiques liées à l'agonie de la mère à l'hôpital et des récits d'enfance, comme des séances de psychanalyse - sans oublier les scènes oniriques -, se présente non seulement dans des rapports d'analogie entre ces scènes, mais aussi, le plus souvent, sous les mêmes modes et temps verbaux. Cet aspect du texte, tout en donnant la sensation, d'une certaine façon, d'enchaîner des temporalités lissées par mise en équivalence à travers l'usage du présent, tend en réalité à renforcer les aspérités temporelles et à rendre les liens entre ces composantes hétérogènes plus complexes. Les occurrences du procédé sont nombreuses. Dans l'exemple qui suit, le procédé de la continuité temporelle au présent s'inscrit dans le creuset de la stratégie narrative explicitée auparavant, des sauts de temporalité par feuilletage des temps narrés. Alors que le narrateur est en train de rapporter un souvenir douloureux et fondateur de ses rapports à la mère - celle-ci est sollicitée pour raconter une histoire du soir, alors que le père, normalement maître d'œuvre de ces contes, est absent -, la suite du texte nous replonge dans la diégèse textuelle sans que le lecteur soit à même de le saisir avant d'avoir avancé dans sa lecture :

14 Il s'agit d'une mesure de l'anachronie d'un point de vue strictement fictionnel.

15 Gérard Genette, Discours du récit, op. cit., p. 78. 
[...] Nous insistons très cruellement, maman se navre. Nous sentons bien qu'elle a envie de pleurer, mais nous ne pouvons pas nous empêcher de réclamer sur un ton plaintif : Une vraie histoire. J'éprouve encore son chagrin, le froid qui la gagne à nouveau, mais aussi l'impossibilité de rien faire pour éviter l'irréparable malentendu. Car finalement maman se lève et nous dit d'un ton sec d'aller nous coucher. Elle a oublié la prière, nous le lui faisons remarquer, elle se détourne. Est-ce qu'elle va s'en aller sans dire la prière? Non, elle se joint à nous, comme d'habitude, mais sa voix est glacée, elle nous borde, elle nous embrasse rapidement et sort comme quelqu'un qui n'est pas aimé.

Voici Bruxelles et la tache de lumière que la ville fait au milieu des bois et des champs, la longue tache grise qu'elle a fait dans ma vie. Ici, j’ai commencé à me défaire, à Paris a commencé l'opération de la mort, comme si on passait du gris au noir. Mais à Paris il y avait l'espoir d'une nouvelle naissance, ici rien que la mécanique du désastre et la certitude de la vie fausse. J'ai peur de m’y retrouver, comme dans une ancienne prison. Déjà j'ai l'impression d'étouffer dans les interminables couloirs de l'aérogare et je me presse pour en sortir. (D98, p. 54-55)

Alors que la fin du premier paragraphe nous laisse sur une scène d'enfance traumatique, celui qui enchaîne n'indique en rien à quelle époque se situe la scène. "Voici Bruxelles " indique une rupture vis-à-vis de la scène qui précède, mais ce n'est que dans les deux dernières phrases du nouveau paragraphe que les informations, nécessaires au lecteur pour qu'il puisse se repérer, lui sont transmises. La paralipse initiale, soutenue par la forme d'équivalence que génère le présent entre les deux périodes narrées, entend ainsi installer le lecteur dans un rapport métonymique - empathique ? - de déboussolement avec le narrateur, qui est en train de vivre, au présent de la narration, ces hoquets narratifs de l'histoire familiale.

C'est dans un autre texte de L'Écriture à l'écoute que l'on trouve la clé de ce travail de composition narrative. Henry Bauchau y explicite en effet ce qui a motivé une telle mise en forme du récit et l'usage extensif de ce présent de narration, perturbateur de la lecture :

Les années passent, ma mère meurt. Elle, si discrète, lutte farouchement dans le silence pour garder le souffle, elle le perd au bout d'un combat. Pendant la semaine de son agonie, le temps des séances, ce que j'y ai vécu, ce que je n'ai pas pu encore en vivre, m'assaille. Au cours de ces jours et de ces nuits je me sens vivre à la fois dans les deux temps, celui du présent au chevet de ma mère et celui d'un autre présent, perpétué, qui surgit du temps des séances. (EE, p. 112)

Dès lors, pour reprendre un autre titre de Henry Bauchau, on pourrait dire que le récit s'écrit au "présent d'incertitude » ${ }^{\mathbf{1 6}}$ et que c'est grâce à ce nouveau temps, vécu de l'intérieur et non rattaché à une temporalité historique, que le narrateur s'extirpe de la forme d'éternité que semble représenter l'enfance à ses yeux et qui,

16 Le titre dit l'incertitude ressentie par l'auteur, mais on peut aussi l'entendre comme un nouveau temps de conjugaison. Ce " présent d'incertitude "vient renforcer, en ce sens, le flottement que fait subir au texte l'usage du "présent de narration ". 
clairement, l'empêche de s'affirmer en tant qu'adulte. De même, cette élaboration narrative complexe permet la relance, par le truchement de l'écriture, de la temporalité intrinsèque au Moi en perlaboration. Par suite, la reprise des épisodes de l'enfance et de l'adolescence au fil du récit travaille le texte comme le fait un ostinato en musique ${ }^{17}$. Finissant par déborder de partout la chronologie du personnage, l'ostinato en vient à être lui-même débordé, notamment en raison du stratagème d'écriture $a$-chronique, qui fait déborder, in fine, toutes les temporalités les unes sur les autres. En d'autres termes, la " connivence des temps ", sous les auspices du Grand Temps, en vient à subsumer toute narration relatée au présent de narration, ou, pour mieux dire, offre la possibilité au récit d'outrepasser les catégories temporelles conventionnelles du genre romanesque.

Ainsi, c'est à travers le redéploiement des deux valeurs narratives temporelles du Grand Temps et du présent d'incertitude qu'un Moi, à même les failles et interstices du soi, parvient à se reconstruire par l'écriture et ce, à travers la redistribution $a$-chronique de l'histoire du personnage. Quand bien même il n'y va pas d'une entité au Moi unifié18. Ainsi, les différents membres temporels recomposés dans et par la texture narrative, engendrent, à travers leur réassortiment même, un Moi, certes aux allures disparates, mais enrichi des déchirures mêmes de ses temporalités intérieures, et des réparations qu'opère la langue comme suture. En d'autres termes, l'éparpillement des temporalités narrées donne à lire l'ensemble des différents Mois du narrateur, diffractés, éparpillés, mais recomposés par le travail d'écriture dans $L a$ Déchirure tel le manteau d'Arlequin décrit par Michel Serres dans Le Tiers-Instruit:

Bigarrure composite, faite de morceaux, en haillons ou lambeaux, de toutes tailles, mille formes et couleurs variées d'âges divers, de provenances différentes, mal faufilés, juxtaposés sans harmonie, sans attention portée aux voisinages, reprisés selon les circonstances, à mesure des besoins, d'accidents et de contingences, montre-t-elle une sorte de mappemonde, la carte des voyages du comédien, comme une valise constellée de marques ?19

Une nuance s'impose, cependant, par rapport à la recomposition du tissu narratif bauchalien : le texte n'est certainement pas "mal faufilé ", ni "juxtaposé sans harmonie ", c'est bien plutôt dans le subtil agencement de la "bigarrure " des matériaux temporels composites que trouve à se déployer l'harmonie d'ensemble du récit.

17 "Ostinato (ital. "obstiné"). Ce terme désigne un élément mélodique ou rythmique plus ou moins court se répétant périodiquement et "obstinément", cependant que d'autres éléments (voix superposées, rythmes simultanés, etc.) se modifient simultanément. La basse répétée, ou basse contrainte (basso ostinato), qui est un dessin mélodique de base de quatre à huit mesures se répétant indéfiniment est un cas particulier d'ostinato [...]. On applique aussi le terme d'ostinato à la répétition insistante d'une brève cellule rythmique ou rythmico-mélodique ", dans Marc Vidal (dir.), Dictionnaire de la musique, Paris, Larousse, "In Extenso ", 2005, p. 1048-1049, souligné dans le texte. C'est dans le deuxième sens que je rapproche le rythme narratif de La Déchirure de celui de l'ostinato musical.

18 À l’image du frère aîné tel que se le représente le narrateur, par exemple.

19 Michel Serres, Le Tiers-Instruit (1991), Paris, Gallimard, "Folio Essais ", 1992, p. 12. Le manteau d'Arlequin peut être une autre métaphore de l'art de la couture des différents Mois du narrateur comme des temps. 
Pour conclure, il appert que la cohérence de La Déchirure ne pouvait pas se trouver à travers une linéarité des épisodes contés, mais devait prendre corps à travers les différents événements, constitutifs de la vie du narrateur en les rapportant dans leurs failles et déchirures temporelles. Elle révèle, en son principe même, une lecture éclatée et plurielle du Moi narrateur, en devenir dans le cours du récit.

Ainsi, à travers cet ouvrage, Henry Bauchau relance sur le plan littéraire la longue tradition philosophique d'interrogation de la dialectique entre durée et simultanéité. En effet, ce n'est plus la durée chronologique de l'être qui peut imposer son cours à la narration, mais bien la réinscription, à chaque instant de l'écrit, de l'éparpillement des temporalités du devenir du narrateur ${ }^{\mathbf{2 0}}$. Il faut que, pour "renaître ", le temps de la narration " meure " à chaque instant, d'où la stratégie d'interruption des temps narrés les uns par rapport aux autres dans le texte de Bauchau, pour qu'à travers ces proliférations, le Moi narrateur puisse renaître des cendres du temps qui le hantent.

Par suite, ce n'est qu'avec l'achèvement du récit - et non de l'histoire du narrateur qui, elle, ne se clôt pas de manière définitive même si elle s’interrompt -, et à travers la stratégie narrative temporelle mise en jeu tout au long du livre, que la suture réparatrice du Moi narrateur parvient à s'accomplir. L'éparpillement temporel des événements en cours de narration autorise le Moi disséminé du narrateur à se restructurer, grâce au remembrement textuel que les coutures ana-logiques du récit lui ont permis d'opérer.

\author{
Olivier Ammour-Mayeur \\ Sorbonne-Nouvelle - Paris 3
}

20 "Le temps pourra sans doute renaître, mais il lui faudra d'abord mourir. Il ne pourra pas transporter son être d'un instant sur un autre pour en faire une durée. " Gaston Bachelard, L'Intuition de l'instant (1932), Paris, Le Livre de Poche, "Biblio essais », 1994, p. 13. 